Document downloaded from:

http://hdl.handle.net/10251/181058

This paper must be cited as:

Ardid Ramírez, M.; Tortosa, DD.; Martínez Mora, JA. (2019). A Compact Array Transducer for Full Calibration of Underwater Acoustic Detection Neutrino Telescopes. IEEE. 591-595. https://doi.org/10.1109/IOTSMS48152.2019.8939244

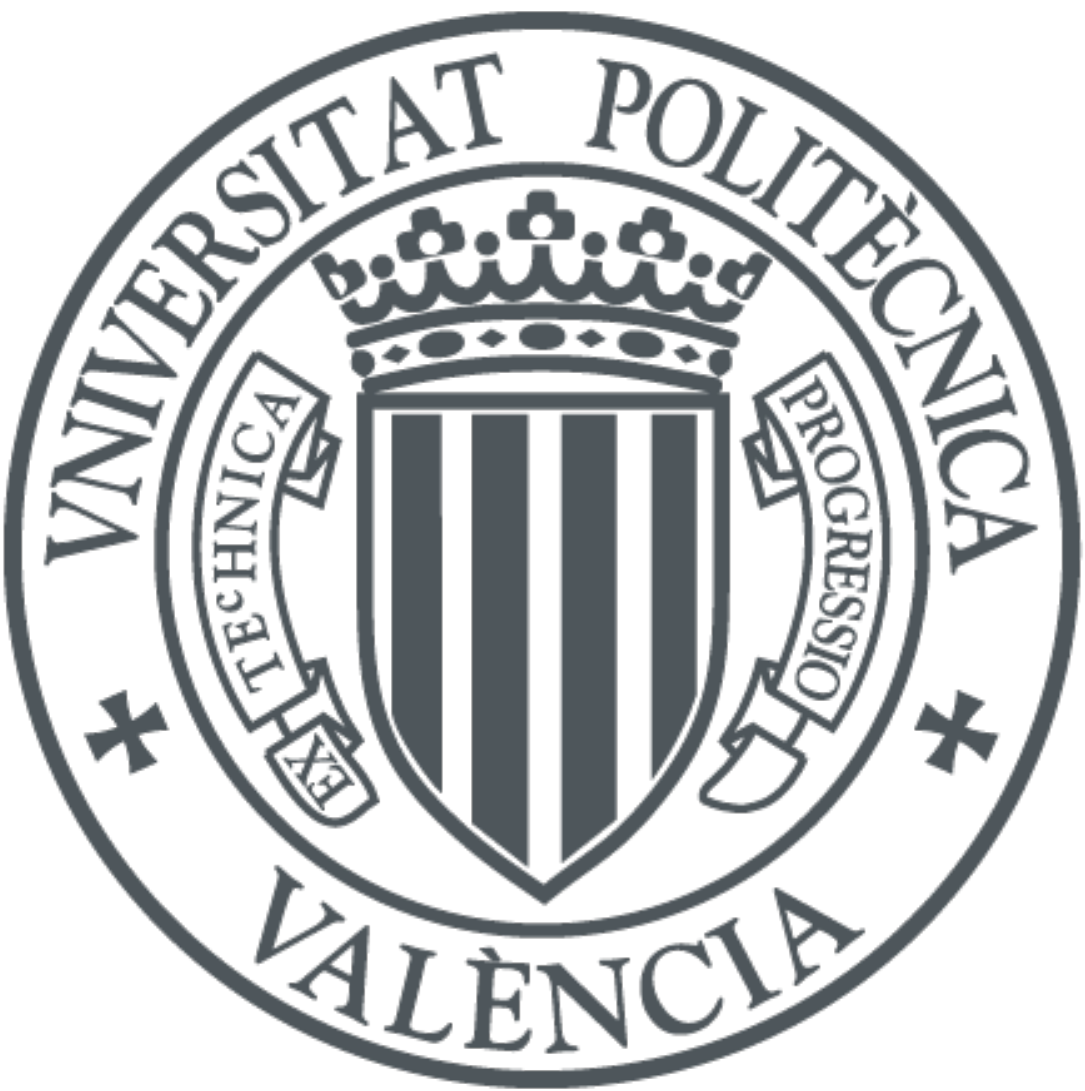

The final publication is available at

https://doi.org/10.1109/IOTSMS48152.2019.8939244

Copyright IEEE

Additional Information 


\section{A Compact Array Transducer for Full Calibration of Underwater Acoustic Detection Neutrino Telescopes}

\author{
Miguel Ardid \\ Institut d'Investigació per a la Gestió \\ Integrada de les Zones Costaneres \\ (IGIC) - Universitat Politècnica de \\ València (UPV) \\ C/ Paranimf 1, 46730 Gandia, València. \\ mardid@fis.upv.es
}

\author{
Dídac D.Tortosa \\ Institut d'Investigació per a la Gestió \\ Integrada de les Zones Costaneres \\ (IGIC) - Universitat Politècnica de \\ València (UPV) \\ C/ Paranimf 1, 46730 Gandia, València. \\ didieit@upv.es
}

\author{
Juan A. Martínez-Mora \\ Institut d'Investigació per a la Gestió \\ Integrada de les Zones Costaneres \\ (IGIC) - Universitat Politècnica de \\ València (UPV) \\ C/ Paranimf 1, 46730 Gandia, València. \\ jmmora@fis.upv.es
}

\begin{abstract}
KM3NeT, the underwater neutrino telescope in the Mediterranean Sea, is a detector under construction. KM3NeT uses Digital Optical Modules (DOMs) to detect neutrinos but there will be a study about the viability to acoustic detection of neutrinos using mainly the acoustic sensors the telescope has for positioning purposes. For this, it is necessary to calibrate and test the acoustic response of the receivers in the detector to determine the sensitivity to detect the neutrino acoustic signal and discriminate it from the environmental background. In this work, the strategy for the calibration of the sensor system using a compact array using three steps (frequency, directivity and neutrino signal-like) is described. Moreover, some R\&D activities and results about the second step (long parametric directive signals) are shown.
\end{abstract}

Keywords- Underwater Acoustics, Calibration, Neutrino Detection, KM3NeT

\section{INTRODUCTION}

The acoustic detection of neutrinos is one of the most promising possibilities to extend the neutrino telescopes to the ultra-high-energy (UHE) range. In fact, having a hybrid submarine telescope (optical-acoustic) in the sea, is the only possibility to extend the energy range from tens of $\mathrm{GeV}$ (optical sensors) up to $10^{12} \mathrm{GeV}$ (acoustic sensors), with a reasonable number of sensors. This technique is based on the acoustic detection of the pressure pulse generated by the heating of the water by the hadron cascade after the interaction of the neutrino. The particularities of the radiated pulse (highdirectivity bipolar pulse with axial symmetry) would allow, on the one hand, to distinguish it from other types of signals much more abundant, and on the other hand, to determine the source direction with a few degrees of resolution.

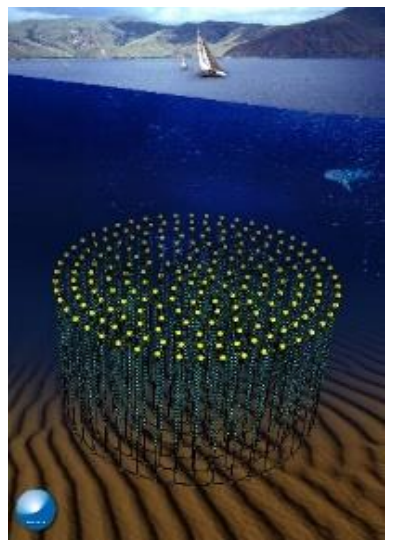

Fig. 1. Schematic view of one structure of DUs in KM3NeT (credit: KM3NeT Coll.)
KM3NeT [1], which is now under construction, will be the biggest underwater neutrino detector in the world. It is located in the Mediterranean Sea and will use thousands of Digital Optical Modules (DOMs) arranged in vertical structures, called Detection Units (DUs), each one with 18 DOMs. This will form a 3D array of optical sensors (see Fig. 1) to detect neutrinos through the Cherenkov light emitted by the relativistic particles produced in the interaction.

Each DOM has installed a piezo-ceramic transducer (acoustic receiver). Additionally, there will be about a hundred of hydrophones on the sea bottom. The main purpose is the use for the Acoustic Positioning System (APS) [2], but since they are continuously monitoring the sound in the $1-50 \mathrm{kHz}$ frequency range needed for the acoustic neutrino detection, they will be used for it as well. The acoustic detection of particles is quite tricky and to discriminate it from background is not trivial $[3,4]$, for this reason it is very important to have a sophisticated calibration protocol that enables to determine the response of the acoustic system in terms of the frequency response, directivity response, and neutrino-like signal sensitivity. The compact array acoustic emitter calibrator we are developing will be able to generate signals in the threestep calibration scale. The prototype will be tested by sending acoustic signals to KM3NeT from a ship [5]. Later on, some of these elements could be installed in the telescope for the systematic use.

In this paper, the compact array developed to fulfil the three calibration scales for the use of the acoustic receivers in KM3NeT for acoustic neutrino detection is described. Next, we present the procedure and utility for the different steps: linear emission ( $1^{\text {st }}$ step), long parametric directive signals ( $2^{\text {nd }}$ step) and bipolar parametric directive signal ( $3^{\text {rd }}$ step). More details will be given to the recent experimental results on the performance of the compact array for the $2^{\text {nd }}$ step.

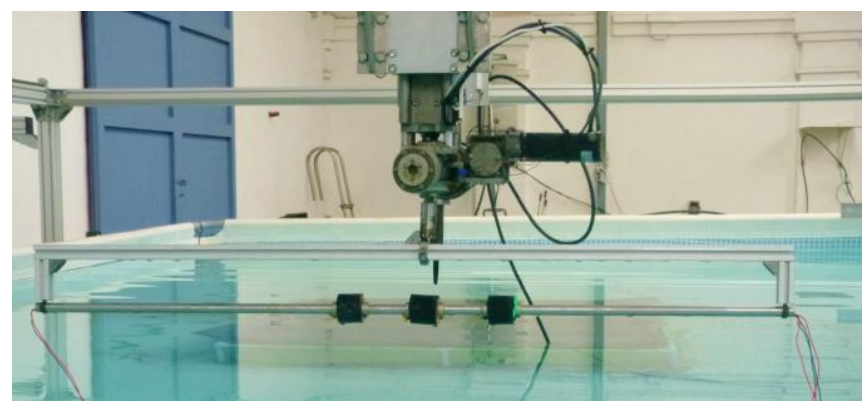

Fig. 2. Picture of the compact array transducer prototype during tests in the lab. 
The paper will be structured as follows. First, a summary of the previous related works about the idea of producing an array of emitters to reproduce a neutrino's acoustic signal, and on the first efforts and results in previous arrays developed.

Next, the design and development of the last prototype is explained in section II.

The parametric effect and the creation of sweep and bipolar pulse parametric signals are described in section III.

Then, the full calibration process proposed for the acoustic sensors in KM3NeT is showed in section IV.

At the end, some conclusions and future steps are discussed.

\section{Related work}

$\mathrm{KM} 3 \mathrm{NeT}$ is the next generation of neutrino detector telescope in the Mediterranean Sea. ANTARES is the existing, i.e. previous neutrino detector in the Mediterranean site [6]. It is operative for more than 10 years. In this type of detectors there are installed acoustic sensors that form an Acoustic Position System to monitor the optical modules that form the telescope. A future idea is to detect acoustic signals from neutrino's interaction with water directly through the detection of the thermoacoustic pulse produced. In any case, the acoustic sensors should be calibrated. For this proposal the idea is to develop an acoustic array to emit a known acoustic signal from a ship to the detector and calibrate the acoustic system.

The first prototype of this kind of calibrator arrays had 8 emitters along $8 \mathrm{~m}$ of length. It was designed according to simulations with the purpose of generating a coherently acoustic bipolar pulse at $23 \mathrm{kHz}$ using a phased emitter array. In the last step to validate it, it was tested in ANTARES during a sea campaign, but due to some technical problems during the campaign the result were not conclusive [7].

The second array designed was a compact array with 3 emitters along $40 \mathrm{~cm}$ of length. This prototype included a specific electronic part to obtain enough power for parametric generation [8]. It tried to reproduce the acoustic neutrino's signal too, but using parametric techniques. It was tested for large distances $(\sim 112 \mathrm{~m})$. It was operative in linear emissions, but it presented low efficiency of parametric generation and a misalignment in the emitters [9].

The third prototype of array is presented here. The successful deployment and operation of this compact array transducer was demonstrated from studies with one emitter element and some simulations [10]. The prototype array is deployed with 3 emitters for first studies, but the final idea is building it with 5 elements. In this proceeding, the array and its usability for characterization of acoustic sensors in $\mathrm{KM} 3 \mathrm{NeT}$ is presented.

\section{The COMPACt ARray Transducer PrototyPe}

The acoustic receivers in KM3NeT have a lot of functions, the most important is for positioning the DOMs, but there are other functionalities, such as the detection and monitoring of marine life, the boat traffic noise, etc. Other possible functionality under study is the detection of UHE neutrinos acoustically. The compact array developed was indeed motivated so to have a tool that could improve the capabilities of first deep-sea acoustic detection neutrino telescopes and check the viability of the technique.

The compact array transducer prototype design was described in a $\mathrm{PhD}$ thesis [5]. Its main objective is to emit acoustic signals to calibrate the acoustic receivers installed in the underwater neutrino telescope KM3NeT in the two sites: ORCA in France at $2400 \mathrm{~m}$ depth, and ARCA in Italy at 3500 $\mathrm{m}$ depth [1]. The particularly of this array is that it is able to emit directive signals with cylindrical axial symmetry similar to the ones produced after a UHE neutrino interaction. This is achieved making use of the parametric effect that allows to reduce the size of the emitter array to $\sim 1 \mathrm{~m}$, thus, compact enough to ease operation in the sea, managed from a vessel.

The array is designed with 3 to 5 emission elements with a distance separation of $14 \mathrm{~cm}$ and connected in parallel. For the moment, a prototype is assembled with 3 elements (see Fig.2). The resonance frequency of their elements is around $380 \mathrm{kHz}$ [11] and this frequency is used for the primary beam to generate the parametric signals. The Transmitting Voltage Response (TVR) of the array is presented in Fig.3.

Since the array has about $160 \mathrm{~dB}$ re $1 \mu \mathrm{Pa} / \mathrm{V} @ 1 \mathrm{~m}$ of transmission and connected to a high-power amplifier, it is possible to emit acoustic signals with amplitude larger than 10 $\mathrm{kPa}$, so with sufficient energy to produce a noticeable parametric effect able to produce the directive signals at low frequency that we aim.

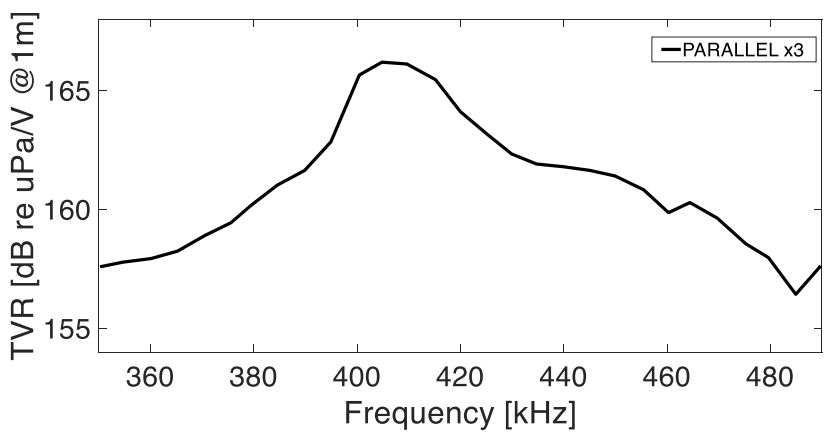

Fig. 3. TVR of array transducer prototype

\section{THE PARAMETRIC EFFECT}

The parametric effect is a non-linear physical phenomenon that appears in the propagation of intense waves. In acoustics, it may result in the appearance of 'new' frequencies that were not present in the signal emission during its propagation. It corresponds to combinations of existing frequencies, such as sum, difference frequencies and harmonics.

This effect permits to produce a differential frequency $\left(2^{\text {nd }}\right.$ beam) between two near tones of high frequencies ( $1^{\text {st }}$ beam) during the propagation of the high-intensity wave ( $1^{\text {st }}$ beam). The parametric acoustic technique has been used to produce directive low frequency signals $[12,13]$. The main advantage of using the parametric effect is obtaining low frequencies signals with high directivity. For large distances, the low frequency signal $\left(2^{\text {nd }}\right.$ beam $)$ is quite clean because the high frequency signal ( $\left(1^{\text {st }}\right.$ beam) is absorbed earlier.

The parametric effect was first derived by Westervelt and then applied to transient signals by Moffet and Mello (1979) that determine the pressure for the secondary beam depending 
of the power of the primary beam emitted signal and its envelope (equation 1):

$$
p(x, t)=\left(1+\frac{B}{2 A}\right) \frac{P^{2} S}{16 \pi \rho c^{4} \alpha x} \frac{\partial^{2}}{\partial t^{2}}\left[E\left(t-\frac{x}{c}\right)\right]^{2}
$$

Where $p$ is the pressure of 2 nd beam in $x$ position at time $t, \frac{B}{A}$ is the non-linear medium parameter, $P$ is the pressure of the emitted signal ( $1^{\text {st }}$ Beam $), S$ the surface of emitter transducer, $\rho$ the medium density, $c$ the propagation sound velocity in the medium, $\alpha$ the sound absorption medium coefficient and $E$ the envelope function of the emitted signal.

It is also possible to predict the secondary beam behavior for this compact array transducer using the parametric study of Berktay and Meahy (1974) [14].

This work presents 3 different techniques to apply in the characterization of underwater neutrino's telescope, two of which uses parametric signals $\left(2^{\text {nd }}\right.$ and $3^{\text {rd }}$ step, a parametric sweep signal and a parametric bipolar pulse) and their creation process is described then.

\section{A. Parametric Sweep Signal}

This signal consists in a sine signal at resonance frequency of the emitter used as carrier signal ( $1^{\text {st }}$ beam of parametric effect) modulated with a linear sweep signal of low frequency ( $2^{\text {nd }}$ beam) using DSB (Double Sideband) modulation. The signal emitted is the $1^{\text {st }}$ beam in the propagation. The parametric effect from this signal produces a $2^{\text {nd }}$ beam during its propagation through the frequency difference. The pressure estimation for $2^{\text {nd }}$ beam can be obtained by the second derivative of the square envelope of the emitted signal.

The $2^{\text {nd }}$ beam produced by parametric sweep signal is described like in equation 2.

$$
\begin{aligned}
p_{d} \sim \frac{\partial^{2}}{\partial t^{2}} E^{2}=4 \pi\left\{\frac{\left|f_{2}-f_{1}\right|}{T} \cdot \sin \left[4 \pi \cdot t\left(\frac{\left|f_{2}-f_{1}\right|}{T} \cdot t+f_{1}\right)\right]\right. \\
+2 \pi\left(2 \frac{\left|f_{2}-f_{1}\right|}{T} \cdot t+f_{1}\right)^{2} \\
\left.\cdot \sin \left[4 \pi \cdot t\left(\frac{\left|f_{2}-f_{1}\right|}{T} \cdot t+f_{1}\right)+\frac{\pi}{2}\right]\right\}
\end{aligned}
$$

Where $p_{d}$ is the estimation pressure for differential frequency signal $\left(2^{\text {nd }}\right.$ beam) produced by the parametric effect in the time $t$. The $p_{d}$ is proportional at second derivative of the square envelope of the emitted signal $(E), f_{1}$ and $f_{2}$ are the initial and final frequency in the range of the sweep signal of modulation, $T$ its duration.

\section{B. Parametric Bipolar Pulse}

The acoustic UHE neutrino interaction signature in a fluid medium corresponds to a bipolar pressure pulse with cylindrical symmetry inherited from the shape of the source, that is the hadronic shower produced in the interaction that is contained in a kind of cylinder of a few meters length and a few $\mathrm{cm}$ diameter that produced the pulse thermosacoustically. Thus, this acoustic signal is really singular because it contains a large low frequency range (approximate between 2 to $50 \mathrm{kHz})$ and it is extremely directive $\left(\sim 1^{\circ}\right)[15]$.
An easy way to implement a bipolar pulse mathematically is using the first derivative of a Gaussian function $(g(x)$, equation 3):

$$
g(x)=\frac{1}{\sigma \sqrt{2 \pi}} e^{-\frac{1}{2}\left(\frac{(x-\mu)}{\sigma \sqrt{2}}\right)^{2}}
$$

Where $\sigma$ is the standard deviation of the distribution, which is centered in $\mu$ position.

So, to generate such a bipolar pulse parametrically we need to use as envelope the second integral of the pulse corresponding to the integral of the Gaussian function, and thus the error function. Thus, the signal modulation (envelope) to be used with the carrier signal is obtained by integrating the Gaussian function $\left(g^{\prime}(x)\right.$, equation 4$)$ :

$$
g^{\prime}(x)=\frac{1}{2}\left(1-\frac{2}{\sqrt{\pi}} \int_{\frac{x-\mu}{\sigma}}^{\infty} e^{t^{2}} d t\right)
$$

The creation of parametric bipolar pulse is presented in Fig.4(a), where is shown in black the envelope of the signal emitted. In Fig.4(b) the estimated 2 nd beam produced by parametric effect during its propagation is shown.
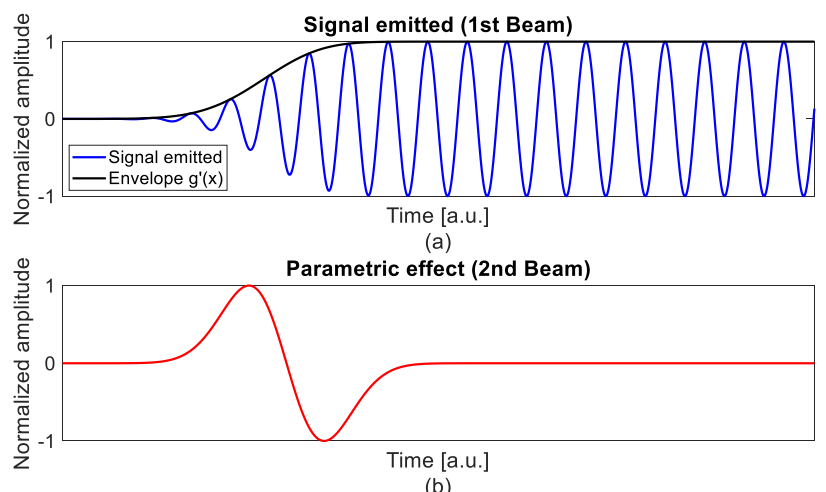

(b)

Fig. 4. Parametric effect estimated (b) in the propagation of parametric bipolar pulse (a)

\section{Full CALIBRATION FOR KM3NET}

As it has already been mentioned, the acoustic receivers in KM3NeT have many purposes for use. Each has some specific characteristics. Moreover, the detection of the UHE neutrino signal and its discrimination from the background is difficult.

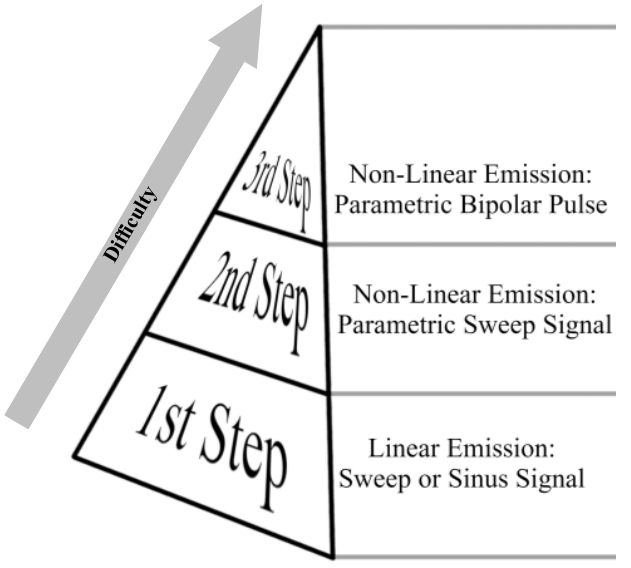

Fig. 5. Strategy for the full calibration with the compact array 
For these reasons the full calibration method for KM3NeT is divided in 3 steps, from more easy and general to the most difficult and specific task (see Fig.5).

The combination of the three in one unique tool, the compact array, not only increase the versatility and functionality of the tool, but also paves the way towards the most difficult task.

The $1^{\text {st }}$ step consists in low-frequency linear emission with the array. The other two steps are directive non-linear emissions using the parametric effect.

The general idea is that using the compact array from a ship (or from a remote operated vehicle) to emit signals from different positions and orientations with respect to the telescope. First, low-frequency non-directive signals will be emitted to study and check directly the reception in the acoustic receivers of the detector ( $1^{\text {st }}$ step). Since it a direct emission, it is quite easy to have a wave with sufficient intensity to reach the telescope and detect it without much trouble. This signal will be used to both calibration of sensors of the telescope and check the propagation of acoustic signals in the sea. Additionally, this kind of signals could also be used to tag and for comparisons with the emission of parametric signals, much more difficult to detect. Then, the use of the parametric signals is foreseen to deal with the low-amplitude signal and directivity expected for the neutrino signature. In a $2^{\text {nd }}$ step, long signals will be used, such as the parametric sine sweep signal. The advantage of this step is that, we can detect the signal easier thanks to the use of signal processing techniques. Additionally, a much clear study can be done, such as the frequency dependence, so obtaining a much better and complete understanding of the situation, and how the directivity of the source is linked to the response of the different sensors of the telescope. Finally, the most difficult part can be done by emitting the bipolar parametric pulse $\left(3^{\text {rd }}\right.$ step), that is fully simulating the acoustic neutrino's signature and calibrating the whole system for this aim and testing the ability to discriminate this kind of signals from the environmental noise.

\section{A. Linear Emission ( $1^{\text {st }}$ step)}

The compact array allows to emit sine and sweep signals directly in low frequency. The TVR is about $135 \mathrm{~dB}$ re 1 $\mu \mathrm{Pa} / \mathrm{V} @ 1 \mathrm{~m}$ in the $10-100 \mathrm{kHz}$ region. Then, with appropriate electronics it is quite straightforward to get $180-190 \mathrm{~dB}$ re 1 $\mu \mathrm{Pa} @ 1 \mathrm{~m}$, and thus capable to reach the detector a few $\mathrm{km}$ away with positive signal-to-noise ratio. Therefore, it is possible to use these linear emissions for check the status of the acoustic receiver and get knowledge about the propagation of signals in situ. Moreover, signals used for the acoustic positioning system of KM3NeT could be done, benefiting from the procedures and protocols for detecting these signals at the same time of being an independent cross-check for that system.

The characteristic of this step is that the emission is for all detector because the linear emission in low frequencies produces a non-directivity emission, and thus, an accurate orientation of the array with respect to the telescope is not needed.

\section{B. Long Parametric Directive Signal ( $2^{\text {nd }}$ step)}

The first non-linear emission to calibrate the acoustic receivers in $\mathrm{KM} 3 \mathrm{NeT}$ is considered using a long parametric directive signal. A long signal provides less difficulty on detection when the signal is received thanks to signal processing techniques, such as cross-correlation. In this context, a long duration signal (of the ms order) is considered.

The main objective of this step is to determine the response of the telescope to very directive (a few degree) signals with cylindrical symmetry [11]. This allows the calibration of the different sensors in the $1-50 \mathrm{kHz}$ region but including the directivity component.

In this $2^{\text {nd }}$ step of the characterization, a parametric sweep signal is designed for the emission. The carrier sine signal is $380 \mathrm{kHz}$ (resonance frequency of the elements in the array) with $1 \mathrm{~ms}$ length. This duration permits a good transition between frequencies, especially for the low frequencies, in the envelope sweep signal. The frequencies of the line sweep signal are between 5 to $25 \mathrm{kHz}$, to produce a $2^{\text {nd }}$ beam like sweep signal between 10 to $50 \mathrm{kHz}$ (the most important frequency range for the acoustic neutrino signal). The parametric signal in reception and its frequencies are show in Fig.6.
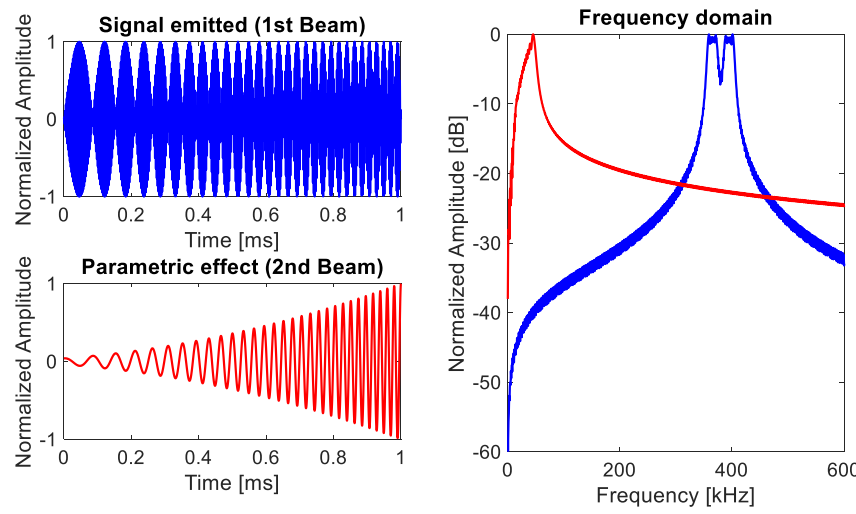

Fig. 6. Long parametric sweep signal emitted (a), the $2^{\text {nd }}$ beam estimated (b) and its Fast Fourier Transform (c)

For analyzing the reception of the large parametric sweep signal in the lab, it will be necessary a Band-Pass filter because the received signal contains both signals mixed $\left(1^{\text {st }}\right.$ and $2^{\text {nd }}$ beams). The $2^{\text {nd }}$ beam amplitude is expected to have a substantial decrease in amplitude with respect the $1^{\text {st }}$ beam reception, $20 \mathrm{~dB}$ in the frequency domain approximately.

Using this signal is possible to characterize the low frequencies in a specific sector, and by combining the information of all the sensors determine the directivity of the source, and thus being able to discriminate the signal from other more frequent less-directive acoustic signals. This information is then critical to improve the performance of the facility and determine the sensitivity.

\section{Bipolar Parametric Directive Signal ( $3^{\text {rd }}$ step)}

For a final characterization of the receivers to the acoustic neutrino's detection and test the viability of the technique is essential to be able to reproduce its acoustic signature. with this aim, a parametric bipolar pulse is created using the compact array. Similarly to the parametric sweep signal, the carrier signal is a sine with resonance frequency of the emitter element, but in this case the envelope signal is the integral of the Gaussian function $\left(g^{\prime}(x)\right)$. In this case, the parametric effect will be produced by the rise of the gaussian function integral. It presents the problem that the amplitude of the signal starts with zero, increases rapidly, but then it remains constant without decreasing to zero. To deal with this, it is 
necessary to produce another decrease transition, that, in turn, generates another acoustic parametric pulse, but inversed. To distinguish them, there must be sufficient time between the parametric pulses. Since the bipolar pulses are of short duration, a $1 \mathrm{~ms}$ length for the total emitted signal seems convenient for both in terms of the energy and in term of analysis resolution.

About the acoustic pulse produced by parametric effect, it is designed by a gaussian increase transition with $2.5 \mu \mathrm{s}$ for sigma parameter (standard deviation) and it is centered at 15 $\mu$ s from the initial time ( $\mu$ parameter). Similarly, the decrease transition is centered $15 \mu \mathrm{s}$ from the end of the signal with 2.5 $\mu \mathrm{s}$ of sigma.

The estimated bipolar pulse received is shown in Fig.7.
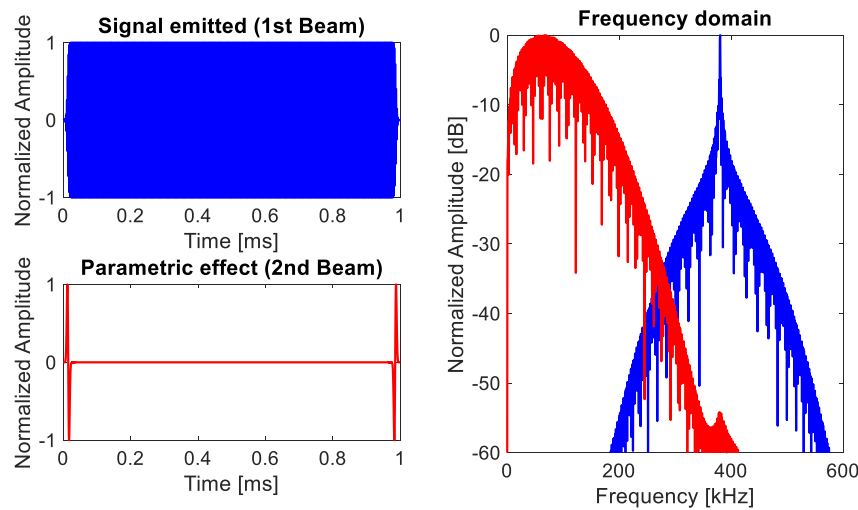

Fig. 7. Bipolar parametric directive signal emitted (a), the $2^{\text {nd }}$ beam estimated (b) and its Fast Fourier Transform (c)

Another thing to keep in mind is the low amplitude of the neutrino acoustic pulse, for this its detection is so difficult and requires a detailed analysis of the recorded signals for distinguishing it from the environmental noise.

\section{CONCLUSIONS AND FUTURE STEPS}

In this paper we have introduced the compact parametric array developed to fulfil the different calibrations for acoustic neutrino detection in deep-sea telescopes. It has been shown that the combination of the three modes of operation: linear emission ( $1^{\text {st }}$ step), long parametric directive signals ( $2^{\text {nd }}$ step) and bipolar parametric directive signal ( $3^{\text {rd }}$ step) allows not only the calibration of the sensors but determining the whole response of the telescope for neutrino-like signals and thus is a very useful tool to both improve the sensitivity of the telescope and test the viability and limitations of the technique.

\section{Future Steps}

The next step is doing some test in situ so to check the emission and reception for all cases, so to have a complete characterization of the array and evaluate its feasibility as in situ calibrator.

Before that, the electronics of the array concerning the amplification of the signal for the parametric use should be improved so being able to produce high-level acoustic signals able to reach large distances $(\sim \mathrm{km})$ with enough intensity for being detected by the sensors of the telescope.

\section{ACKNOWLEDGMENT}

We acknowledge the financial support of the Spanish Plan Estatal de Investigación, ref. PGC2018-096663-B-C43 (MICINN/FEDER)

\section{REFERENCES}

[1] S.Adrián-Martínez, et.al. "Letter of intent for KM3NeT 2.0" J. Phys. G: Nucl. Part. Phys, vol. 43 (8), pp. 084001, 2016. DOI: 10.1088/0954$3899 / 43 / 8 / 084001$

[2] S. Viola, et.al. "Acoustic positioning system for KM3NeT" Proceedings of Science. vol. 236 ICRC2015 pp. 1169, 2016. DOI: 10.22323/1.236.1169

[3] A. Aguilar et al., "AMADEUS - The acoustic neutrino detection test system of the ANTARES deep-sea neutrino telescope". Nuclear Instruments and Methods in Physics Research Section A Accelerators Spectrometers Detectors and Associated Equipment. vol. 626, pp. 128 - 143, 2011. DOI: $10.1016 /$ j.nima.2010.09.053

[4] M. Ardid. "Calibration in acoustic detection of neutrinos". Nuclear Instruments and Methods in Physics Research Section A Accelerators Spectrometers Detectors and Associated Equipment. vol. 604, pp. 203 $-207,2009$. DOI: $10.1016 /$ j.nima.2009.03.071

[5] M. Saldaña-Coscollar. "Acoustic System Development for Neutrino Underwater Detectors" PhD Thesis.Universitat Politècnica de València, June 2017. DOI: 10.4995/Thesis/10251/85981.

[6] M. Ageron et al., "ANTARES: the first undersea neutrino telescope". Nuclear Instruments and Methods in Physics Research Section A Accelerators Spectrometers Detectors and Associated Equipment. vol. 656, pp. 11-38, 2011. DOI: 10.1016/j.nima.2011.06.103

[7] W. Ooppakaew, S. Danaher and M. Saldana, "Acoustic Array Calibration and Signal Processing for UHE Neutrinos Generation" IEEE Eighth International Conference on Mobile Ad-Hoc and Sensor Systems, València, 2011, pp. 910-915. DOI: 10.1109/MASS.2011.109

[8] Llorens, C. D., Ardid, M., Sogorb, T., Bou-Cabo, M., Martínez-Mora, J. A., Larosa, G., \& Adrián-Martínez, S. "The sound emission board of the KM3NeT acoustic positioning system" Journal of Instrumentation. vol. 7(1), p. C01001, 2012. DOI: 10.1088/1748-0221/7/01/C01001

[9] S. Adrián-Martínez, M. Ardid, M. Bou-Cabo, G. Larosa, C.D Llorens, J.A. Martínez-Mora, "A compact acoustic calibrator for ultra-high energy neutrino detection", Nuclear Instruments and Methods in Physics Research Section A: Accelerators, Spectrometers, Detectors and Associated Equipment, vol. 725, pp. 219, 2013. DOI: 10.1016/j.nima.2012.11.142

[10] Saldaña, M.; Llorens, C.D.; Felis, I.; Martínez-Mora, J.A.; Ardid, M. "Transducer Development and Characterization for Underwater Acoustic Neutrino Detection Calibration". Sensors vol. 16, pp. 1210, 2016. DOI: $10.3390 / \mathrm{s} 16081210$

[11] D. D.Tortosa, et.al. "A Compact Transmitter Array to Reproduce the Acoustic Signature of Neutrino in Water". Proceedings, vol.4(4) ECSA5, 2019. DOI: 10.3390/ecsa-5-05748

[12] M. Campo-Valera, et.al. "Underwater Communication Using Acoustic Parametric Arrays". Proceedings, vol.2(3) ECSA4, 2018. DOI: 10.3390/ecsa-4-04907

[13] D. D.Tortosa, et.al. "Acoustic parametric techniques for neutrino telescopes". ARENA2018, June, 2018.

[14] D. D.Tortosa. "Caracterización de un transductor paramétrico para emular el patrón acústico de neutrinos de energía ultra alta como calibrador del telescopio de neutrinos KM3Net" Master's Thesis.Universitat Politècnica de València, September 2017.

[15] K, Graf. "Experimental Studies within ANTARES towards Acoustic Detection of Ultra-High Energy Neutrinos in the Deep-Sea" PhD Thesis. Friedrich-Alexander-Universität Erlangen-Nürnberg (FAU), June 2008. DOI: 10.4995/Thesis/10251/85981. 